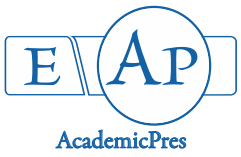

Benslama O et al. (2021)

Notulae Scientia Biologicae

Volume 13, Issue 2, Article number 10835

DOI: $10.15835 /$ nsb13210835

Research Article

\title{
Antimalarial phytochemicals as inhibitors against COVID-19 ACE2 receptor: Computational screening
}

\author{
Ouided BENSLAMA*, Nedjwa MANSOURI, Rabah ARHAB
}

\author{
Laboratory of natural substances, biomolecules and biotechnological applications, Department of Natural and Life Sciences, Larbi \\ Ben M'Hidi University, Oum El Bouaghi, Algeria; benslama.wided@hotmail.fr (*corresponding author); \\ najwamansouri94@gmail.com; arhabrabah@yahoo.fr
}

\begin{abstract}
Quinine, artemisinin, febrifugine, brusatol, chaparrin tehranolide, glaucarubin, sergeoliden, and yingzhaosu A, nine antimalarial phytochemicals, were the focus of an in-silico analysis aimed at discovering new therapeutic molecules against COVID-19 infection. The screening of these molecules included a molecular docking approach within the Angiotensin-converting enzyme-2 (ACE2) receptor. In addition, drug-likeness, ADMET analysis and pharmacophore mapping have been performed. The result of the docking process was based on the energy binding values as well as the number and type of interactions established with the receptor active site residues, which were compared with those of co-crystallized ligand and chloroquine. Febrifugine showed the most interesting energetic and interactive activities that were closer to the reference molecule and better than those of chloroquine. Whereas artemisinin has produced results that are the closest to those of chloroquine. Similarly, drug-likeness and ADMET analysis have shown that febrifugine and artemisinin check most of the filters and pharmacokinetic properties required for the choice of an effective therapeutic molecule. A pharmacophore model was designed on the basis of a training set consisting of the most relevant molecules; it has one metal ligator cum hydrophobic region cum hydrogen bond acceptor, one hydrogen bond acceptor cum metal ligator and one hydrophobic aromatic ring. This model is proposed to be used for the in-silico discovery of new therapeutic molecules against coronavirus.
\end{abstract}

Keywords: ACE2; antimalarial phytochemicals; COVID-19; molecular docking; pharmacophore; SARS-Cov-2

\section{Introduction}

Coronaviruses are a family of viruses, some of which can infect humans, most often causing mild coldlike symptoms. However, three deadly epidemics have already occurred, including the current Severe Acute Respiratory Syndrome Coronavirus 2 (SARS-Cov-2). SARS-CoV-2 is a new coronavirus identified as the cause of 2019 coronavirus disease (COVID-19) which started in Wuhan, China in late 2019 and has spread around the world (Gorbalenya et al., 2020). At present, over 136 million cases of infection have been officially diagnosed over the world (Dong, 2020). As the SARS-Cov-2 coronavirus pandemic spreads rapidly around the world, research is mobilizing for knowledge on this virus, on the disease it causes, as well as the discovery of effective drugs against this virus. At present, the World Health Organization WHO has approved the clinical 
trials for Hydroxychloroquine, a derivative of Chloroquine, which is the only proposed treatment for COVID19 known to date (Wang et al., 2020).

Chloroquine is an aminoquinoline known primarily for its antimalarial effect (Foley and Tilley, 1998; Krafts, 2010). In addition, the anti-inflammatory (Macfarlane and Manzel, 1998; Kuznik et al., 2011) and antiviral (Savarino et al., 2001; Vincent et al., 2005; Di Trani et al., 2007) properties of chloroquine are also well known. After given an antiviral activity against SARS-Cov-2 in vitro (Wang et al., 2020; Yao, 2020), the inhibitory activity of the chloroquine has been demonstrated by clinical studies carried out on patients infected with the virus (Gautreta et al., 2020; Huang, 2020). Several hypotheses have been made to explain the mode of action of Chloroquine, but the most relevant of these hypotheses is the finding that chloroquine interferes with the angiotensin-converting enzyme-2 (ACE2) which is the cellular receptor of SARS-Cov, thereby inhibiting the entry of the virus into target cells (Vincent et al., 2005).

Despite the efficacy of chloroquine in curing COVID-19, it should be noted that the clinical tolerance profile includes some concerns about the adverse effects of the molecule such as that of drug interactions (Marmor et al., 2016; Yao et al., 2020), as well as some major risks noted in the case of high doses and long periods of use of the drug and in cases of concomitant renal disease (Marmor et al., 2016).

Because of the severity of the COVID-19 infection, and its rapid spread, it is essential to discover new molecules which are therapeutically effective against SARS-Cov-2 and have fewer adverse effects than Chloroquine. In fact, the Chloroquine is a synthetic molecule derived from Quinine which is a phytochemical compound extracted from the bark of Cinchona trees. Quinine is known for its therapeutic properties and was the first drug used against malaria (Bruce-Chwatt, 1981). In addition, other phytochemicals extracted from different plants have also been traditionally used and they have shown their effectiveness against this form of fever (Mojab, 2012). It therefore seems interesting to us to look at the effectiveness of these molecules against the coronavirus. In this context, this study aims to propose new phytochemical molecules, known for their antimalarial activity, as potential therapeutic agents against COVID-19. For this reason, an in-silico study by molecular docking was carried out on the ACE2 receptor. In addition, a drug-likness and ADMET analysis was conducted on the selected phytochemicals in order to test their pharmaceutical reliability and efficacy. A pharmacophore has been produced and proposed as a model for screening chemical libraries in order to discover other potential therapeutic molecules to treat SARS-Cov infections. Since the onset of the COVID-19 pandemic, some studies have been interested in the contribution of phytochemicals as therapeutic agents for the cure from the coronavirus infection (Aanouz et al., 2020; Abdelli et al., 2020; Joshi et al., 2020; Kumar et al., 2020), but this study is the first report to deal with the screening of antimalarial phytochemicals against the COVID-19.

\section{Materials and Methods}

\section{Preparation of the structure of target protein}

The 3D structure of the ACE2 was obtained from the PDB database (https://www.rcsb.org) with the identifier: ID 1R4L. In order to remove solvent molecules, water and co-crystallized ligands bound to the receptor, UCSF Chimera 1.13 software was used. The structure of the prepared receptor was saved in PDB format.

\section{Preparation of the structure of ligands}

Based on the literature published on phytochemical compounds with antimalarial properties (Mojab, 2012), the quinine, artemisinin, febrifugine, brusatol, glaucarubin, tehranolide, sergeoliden and yingzhaosu A (Table 1) were chosen in addition to chloroquine as ligands for the docking of ACE2 receptor. The canonical SMILES of these compounds were obtained from the PubChem database (https://pubchem.ncbi.nlm.nih.gov). They were used to design the three-dimensional structures of the ligands by UCSF Chimera software. All the structures were saved in Mol2 format for their use in docking process. 
Table 1.2D structures of the studied ligands

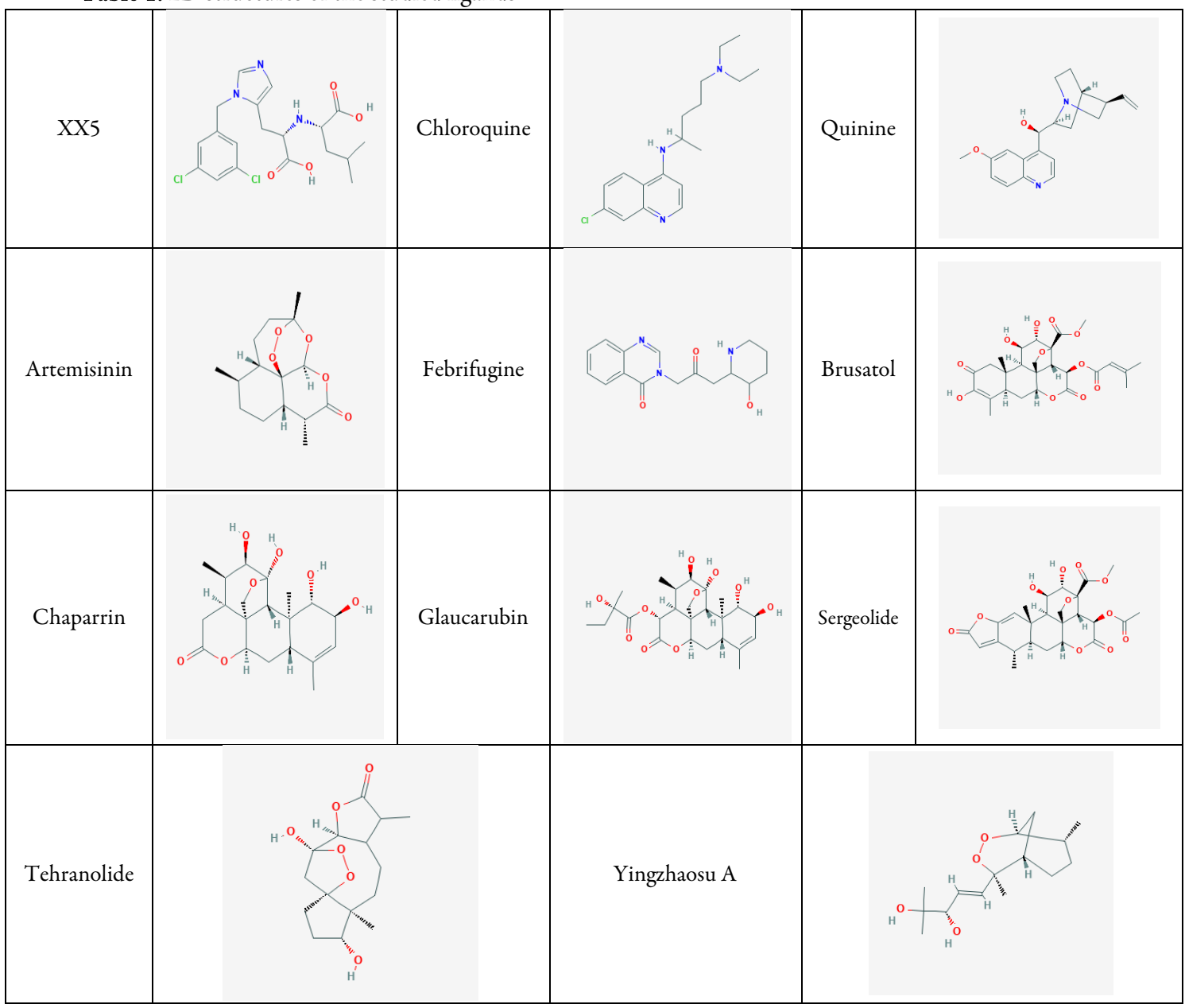

\section{Optimization of target proteins and ligands}

To improve the result of the docking and the receptor-ligand interactions, the PDB coordinates of the target protein and the ligand molecules have been subjected to an optimization of the structure by hydrogen atoms adding and energy minimization in order to release internal stresses, leading to the development of more stable structures. This step was established by Chimera software.

\section{Docking of target protein with ligands}

The docking study aims to determine the best conformation and orientation of a ligand towards a target protein when they are linked to form a stable complex, as well as to assess the binding affinity of the ligand to the target protein (Meng et al., 2011). All the ten ligands have been docked using UCSF Chimera software by using Vina program as a docking engine to find the most promising bond geometry. In order to confirm the validity of the docking process, the operation was initially carried out with the co-crystallized ligand of the ACE2 protein. Thus, the SMILES format of the ligand XX5 was retrieved from PubChem database. Grid centre was determined from the binding site of the ligand XX5. The coordinates of the grid box of docking have been defined: $X=41.71, Y=7.87$ and $Z=26.56$ with the dimensions $28.20 \times 29.60 \times 22.10 \AA$. For all the docking operations carried out, the ligands were considered to be flexible structures while the structure of the target protein was kept rigid. The potential molecules having the best binding affinities for the target receptor are those having a binding energy lower than that of the co-crystallized molecule XX5. 


\section{Analysis of interactions}

To visualize and study the modes of interaction between the ligands and the receptor, the docked conformations were analysed using Discovery Studio 4.0 software, in order to identify the types of bonds between the amino acid residues of the receptor and the ligands. The interactions types are hydrogen bonds, hydrophobic bonds and electrostatic bonds. Distances of the different bonds were also calculated.

\section{Drug-likness activity and ADMET study}

The analysis of drug-likness activity was carried out to study the drug properties of the selected molecules, for this reason, the open source program DruLiTo was used to evaluate the pharmacological significance of the selected molecules based on different rules of drug-likness, namely the Lipinski rule, Veber rule, Ghose filter, $\mathrm{BBB}$ rule, CMC-50 like rule, MDD-Like rule and the Quantitative Estimate of Drug-likeness (QED). The pharmacokinetic study was also established to characterize the absorption, distribution, metabolism, excretion and toxicity (ADMET) of the selected ligands. This study included different factors, namely the Blood Brain Barrier penetration (BBB), Plasma Protein Binding (PPB), Human Intestinal Absorption (HIA), P-glycoprotein transport (Pgp), Caco-2 cell permeability, cytochrome inhibition, carcinogenicity, mutagenesis, cardiotoxicity, hepatotoxicity, bioavailability and synthetic accessibility. The pharmacokinetic properties of the ligands were analysed using the SwissAdme (http://www.swissadme.ch/index.php) AND PreADMET (https://preadmet.bmdrc.kr/toxicity/) servers.

\section{Ligand-based pharmacophore modeling}

A pharmacophore is a model which represents the relative positions of the functional groups of ligands. It can be used for the prediction of the potential interferences between ligands and the active site of a receptor. Once generated, a pharmacophore can serve as a model for screening the inhibitory efficiency of a chemo library for a given receptor. There are different types of pharmacophoric features with specific biological action to identify in a pharmacophore, among these features there is the hydrogen donors and acceptors, hydrophobic atoms and aromatic rings (Tai et al., 2012). In this study, the pharmacophore model was generated using MOE 2009.10 software. The first step consists in the building of a training set database, for this reason, the molecules which gave the best results in docking, drug-likness and ADMET tests were chosen in addition to the cocrystallized ligand (XX5). These molecules are the Chloroquine, quinine, artemisinin and febrifugine. Ligands were energy-minimized using MMFF94x force field with Gradient set 0.00001 . Flexible alignment has been achieved for the training set. The best alignment was then used for the building of the pharmacophore query. A consensus pharmacophore is then established for the chosen alignment. The Polarity-charge-hydrophobicity $(\mathrm{PCH})$ scheme was used. Finally, a set test comprising chloroquine, febrifugine and artemisinin was screened for their pharmacophoric features on the basis of the constructed model.

\section{Results}

\section{Molecular docking and interaction analysis}

In this study, molecular docking was performed with Chemira software, where in addition of the chloroquine, nine phytochemicals were selected for their antimalarial properties and docked within the ACE2 receptor binding site. The docking results were showed that with the exception of sergeolide, yingzhaosu $\mathrm{A}$, all of the tested ligands had a lower binding energy than that of the co-crystallized molecule XX5 (Table 2). The binding energy of the ligand XX5 was $-8.7 \mathrm{kcal} / \mathrm{mol}$, while the lowest binding energy was observed for glaucarubin -10.4. The other compounds have also shown low binding energy values: -10.2 for brusatol, 9.7 for chaparrin, -9.0 for febrifugine, -8.8 for chloroquine, -8.7 for atemisinin, -8.7 for tehranolide and -8.6 for quinine. The interaction of the ACE 2 receptor with the febrifugine and the artemisinin is shown in Figure 1. 
Table 2. Docking result of the studied ligands with ACE2 receptor

\begin{tabular}{|c|c|c|c|c|c|}
\hline & $\begin{array}{l}\text { Binding energy } \\
(\mathrm{Kcal} / \mathrm{mol})\end{array}$ & Hydrogen interactions & Hydrophobic interactions & Electrostatic interactions & Total \\
\hline \multirow[t]{2}{*}{$\tilde{x}$} & \multirow[t]{2}{*}{-8.7} & 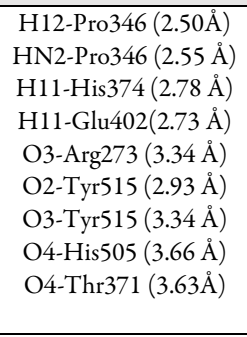 & $\begin{array}{c}\text { Cl2-Phe274 }(5.46 \AA) \\
\text { Cl2-Trp271 }(4.57 \AA) \\
\text { Cl2-Leu503 }(5.49 \AA) \\
\text { C3-Tyr510 }(3.73 \AA) \\
\text { C1-Tyr510 }(3.96 \AA) \\
\text { C1-His505 }(4.11 \AA) \\
\text { C1-Phe504 }(4.94 \AA) \\
\text { C1-His345 (5.13 }) \\
\text { Phe274 }(5.38 \AA) \\
\text { Phe274 }(4.80 \AA)\end{array}$ & H12-Glu375 (2.35 Å) & 20 \\
\hline & & 09 & 10 & 01 & \\
\hline \multirow[t]{2}{*}{ 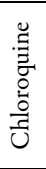 } & \multirow[t]{2}{*}{-8.8} & $\begin{array}{c}\text { H21-Thr371 (2.44 } \AA) \\
\text { N3-Arg273 (3.12 } \AA)\end{array}$ & $\begin{array}{c}\text { C7-Phe274 }(3.51 \AA) \\
\text { Cl-His374 }(4.35 \AA) \\
\text { Cl-Tyr515 }(4.89 \AA) \\
\text { His374 }(5.05 \AA)\end{array}$ & $\begin{array}{c}\text { N1-Glu406 }(3.22 \AA) \\
\text { Glu375 }(4.62 \AA) \\
\text { Arg273 }(3.90 \AA) \\
\operatorname{Arg} 273(4.05 \AA)\end{array}$ & 10 \\
\hline & & 02 & 04 & 04 & \\
\hline \multirow[t]{2}{*}{ 咅 } & \multirow[t]{2}{*}{-8.6} & $\begin{array}{c}\text { HN-Thr371 }(2.14 \AA) \\
\text { H24-Glu406 }(2.28 \AA) \\
\text { O2-Arg518 }(2.86 \AA) \\
\text { C1-Asp368 }(3.73 \AA)\end{array}$ & $\begin{array}{c}\text { C20- Pro346 (4.42 } \AA) \\
\text { Leu370 }(4.71 \AA)\end{array}$ & $\begin{array}{c}\text { N2-Glu406 }(7.05 \AA) \\
\text { N2-Asp367 }(4.91 \AA) \\
\text { Glu375 }(4.88 \AA) \\
\text { Arg273 }(3.97 \AA)\end{array}$ & \multirow[t]{2}{*}{10} \\
\hline & & 04 & 02 & 04 & \\
\hline \multirow[t]{2}{*}{ 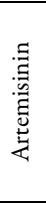 } & \multirow[t]{2}{*}{-8.7} & 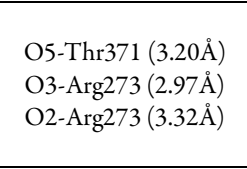 & 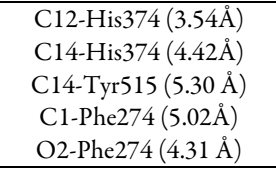 & none & \multirow[t]{2}{*}{10} \\
\hline & & 05 & 05 & 00 & \\
\hline \multirow[t]{2}{*}{ 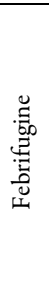 } & \multirow[t]{2}{*}{-9.0} & 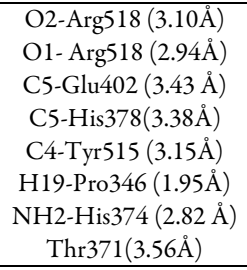 & 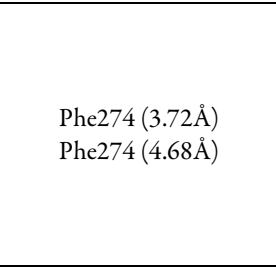 & 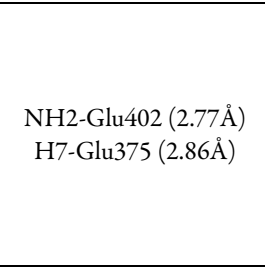 & \multirow[t]{2}{*}{12} \\
\hline & & 08 & 02 & 02 & \\
\hline \multirow[t]{2}{*}{ 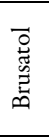 } & \multirow[t]{2}{*}{-10.2} & 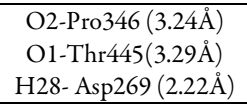 & 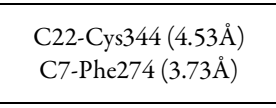 & none & \multirow[t]{2}{*}{05} \\
\hline & & 03 & 02 & 00 & \\
\hline \multirow[t]{2}{*}{ 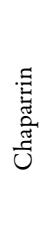 } & \multirow[t]{2}{*}{-9.7} & 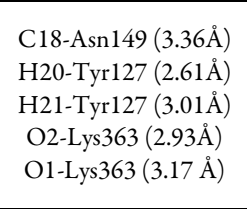 & 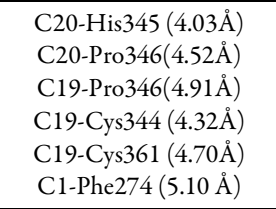 & none & \multirow[t]{2}{*}{11} \\
\hline & & 05 & 06 & 00 & \\
\hline \multirow{2}{*}{ 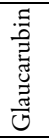 } & \multirow[t]{2}{*}{-10.4} & $\begin{array}{c}\text { O3-Lys363 (3.24 } \AA) \\
\text { H36-Thr371 (2.26 } \AA)\end{array}$ & $\begin{array}{l}\text { C23-Pro346 }(4.12 \AA) \\
\text { C1-Phe274 (3.92 } \AA) \\
\text { C25-Ala153 (3.67 } \AA)\end{array}$ & none & \multirow[t]{2}{*}{05} \\
\hline & & 02 & 03 & 00 & \\
\hline \multirow[t]{2}{*}{ 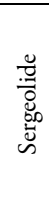 } & \multirow[t]{2}{*}{-6.6} & $\begin{array}{c}\text { O2-Arg514 }(2.71 \AA) \\
\operatorname{Tyr} 510(3.42 \AA)\end{array}$ & 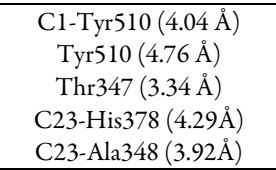 & none & \multirow[t]{2}{*}{07} \\
\hline & & 02 & 05 & 00 & \\
\hline \multirow{2}{*}{ 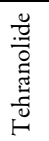 } & \multirow[t]{2}{*}{-8.7} & $\begin{array}{c}\text { H19-Thr445 }(2.84 \AA) \\
\text { H18-Glu406 }(2.85 \AA) \\
\text { O3-Thr371 }(3.32 \AA) \\
\end{array}$ & $\begin{array}{c}\text { C15-Leu370 }(4.80 \AA) \\
\text { C1-Phe274 }(4.67 \AA) \\
\text { Phe274 }(4.21 \AA) \\
\end{array}$ & none & \multirow[t]{2}{*}{06} \\
\hline & & 03 & 03 & 00 & \\
\hline
\end{tabular}




\begin{tabular}{|c|c|c|c|c|c|}
\hline \multirow{2}{*}{ 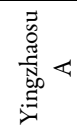 } & \multirow[t]{2}{*}{-7.9} & $\begin{array}{l}\text { O1-Arg518 }(2.96 \AA) \\
\text { O2-Arg273 }(3.02 \AA)\end{array}$ & $\begin{array}{c}\text { C9-His505 }(4.54 \AA) \\
\text { C9-His345 (4.33 } \AA) \\
\text { His374 }(4.88 \AA)\end{array}$ & None & \multirow[t]{2}{*}{05} \\
\hline & & 02 & 03 & 00 & \\
\hline
\end{tabular}

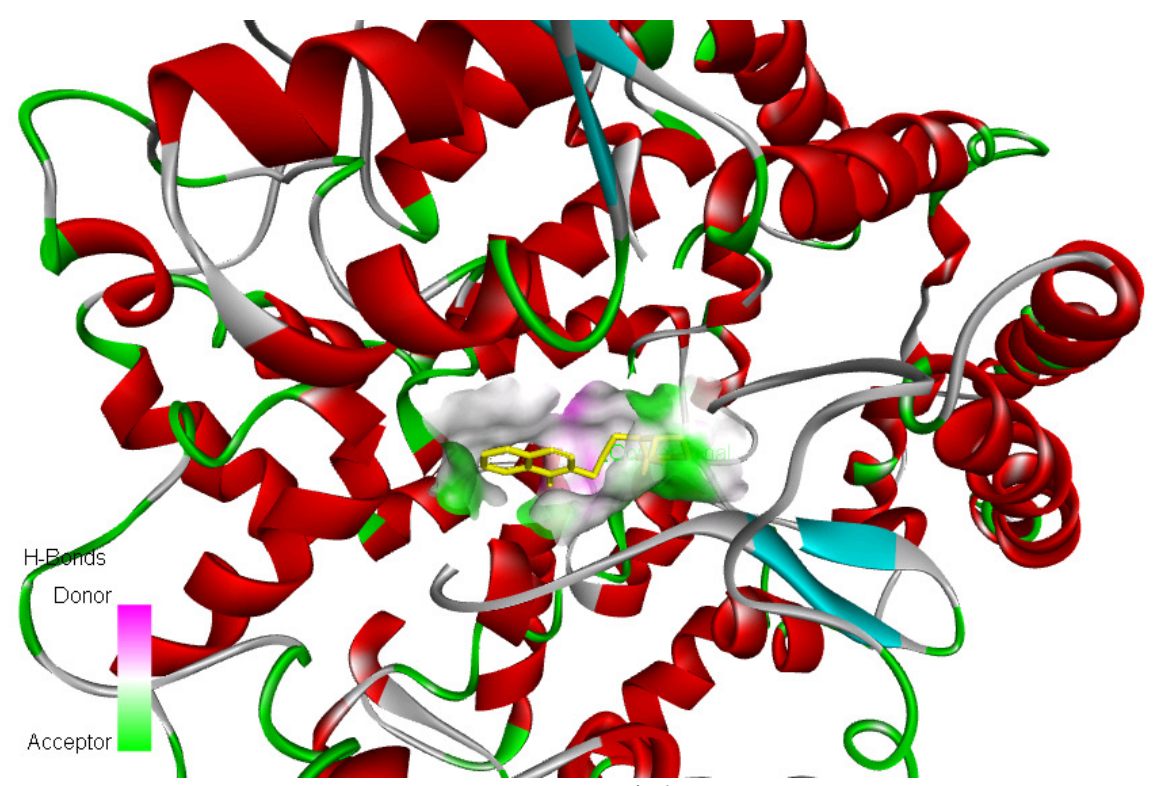

(A)

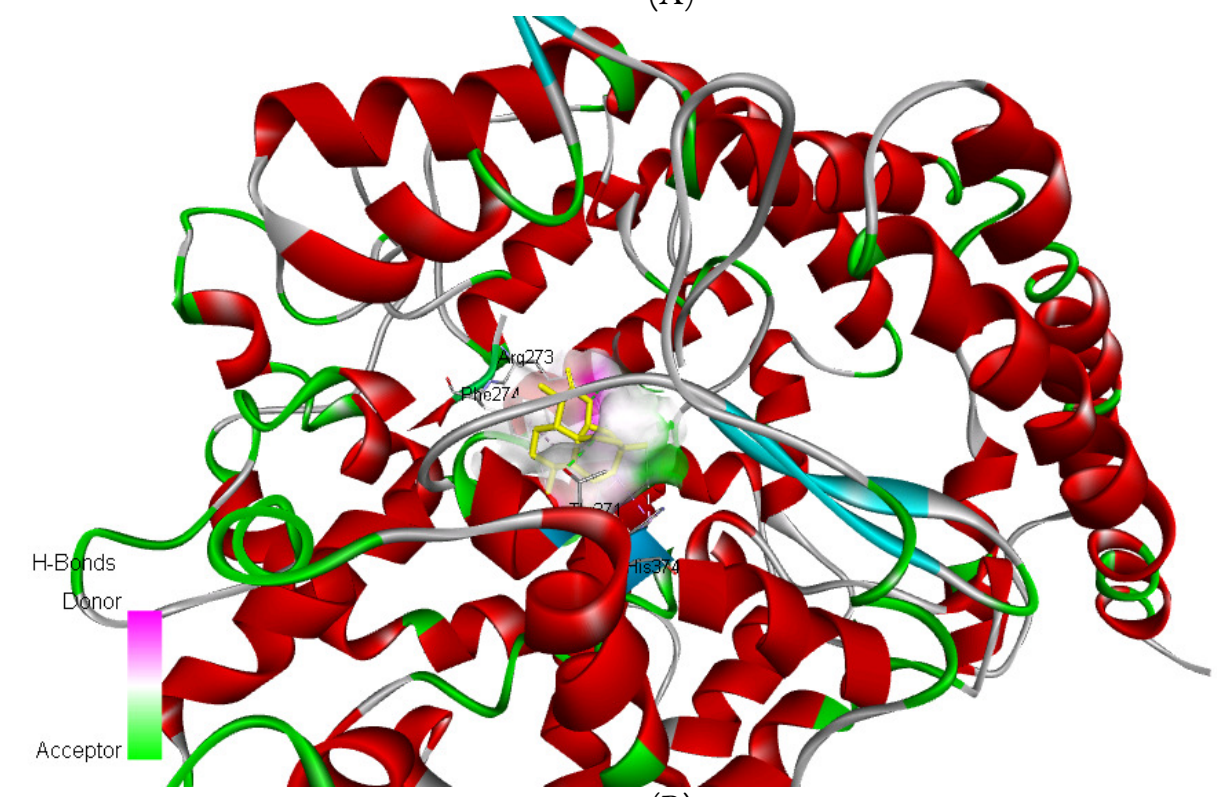

(B)

Figure 1.3D illustration showing the interaction of the ACE 2 receptor with the ligands tested. A; ACE2 receptor complexed with the febrifugine. $\mathrm{B}$; ACE2 receptor complexed with the artemisinin

Regarding the types of interactions identified in the different receptor-ligand complexes and the amino acids involved (Table 2), it was noted that the reference molecule XX5 establishes 20 different interactions within the ACE2 receptor binding site; most of these interactions are hydrogen and hydrophobic bonding type. Chloroquine, artemisinin and quinine were showed a total of 10 different interactions for each. A total of 11 interactions were noted for the chaparrin ligand, while the largest number of interactions was noted in the case 
of the Febrifugine with 12 different interactions, most of which are of hydrogen nature. The other remaining ligands were showed a limited number of interactions: 05 for brusatol, glaucarubin and yingzhaosu A, 06 and 07 different interactions for tehranolide and sergeolide, respectively. The 10 amino acids His374, His 505, His345, Pro346, Glu375, Arg273, tyr515, Thr371, Phe274 and Glu402 appear to be the key residues of the binding pocket of the active site of ACE2 receptor. These amino acids are involved in the reference complex XX5-ACE2. In the case of Chloroquine-ACE2 complex, 6 of these amino acids (Phe274, His374, Thr371, Tyr515, Glu375 and Arg273) participate in the interaction. For the artemisinin-ACE2 complex, the 05 amino acids Thr371, His374, Arg273, Tyr515 and Phe274 contribute to the interaction. Whereas, in the case of quinine, only 4 amino acids among the key residues participate in the quinine-ACE2 complex maintenance, these amino acids are Thr37, Glu375, Pro346 and Arg273. When to Febrifugine, it interacts with 8 key residues, which are His374, Pro346, Glu375, Arg273, tyr515, Thr371, Phe274 and Glu402. In the case of chaparrin and brusatol only the two amino acids Pro346 and Phe274 are present in the receptor-ligand interaction. In addition to these two amino acids, glaucarubin interacts with a third residue which is Thr371 (Figures 2 and 3).

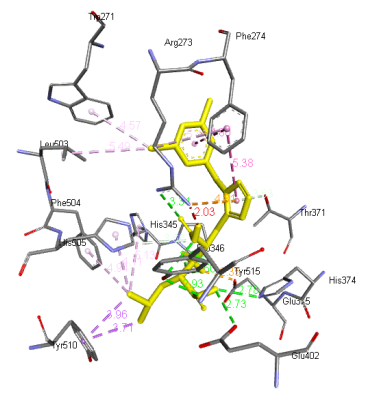

(A)

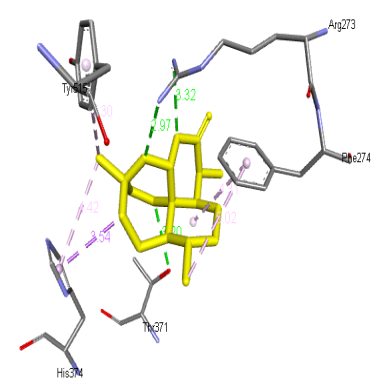

(D)

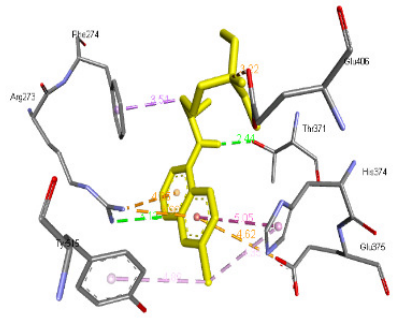

(B)

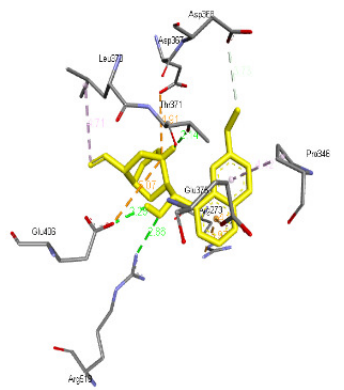

(C)

Figure 2. Depiction of 3D structures showing the interactions between the studied ligands and the receptor ACE2. A1, Interactions between the reference molecule XX5 and ACE2. B1, Interactions between Chloroquine and ACE2. C1, Interactions between Quinine and ACE2. D1, Interactions between Artemisinin and ACE2. E1, Interactions between Febrifugine and ACE2

Drug likness activity and ADMET study

As part of the investigation of the pharmaceutical efficacy of the phytochemicals selected in this study, the results of the drug-likness and ADMET analysis of the various molecules are compared with those of Chloroquine already used as a drug for the treatment of COVID-19 infection. The results of this analysis are collated in Table 3. In complementarity with drug likeness study, the ADMET properties of the studied molecules were tested and their results are grouped in Table 4. 


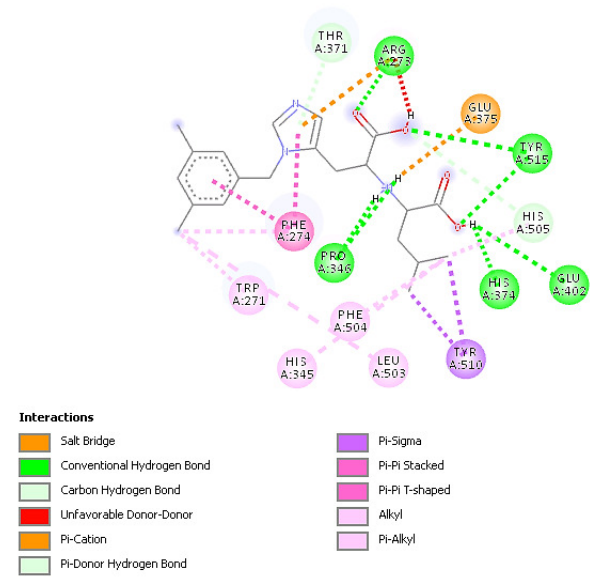

(A)

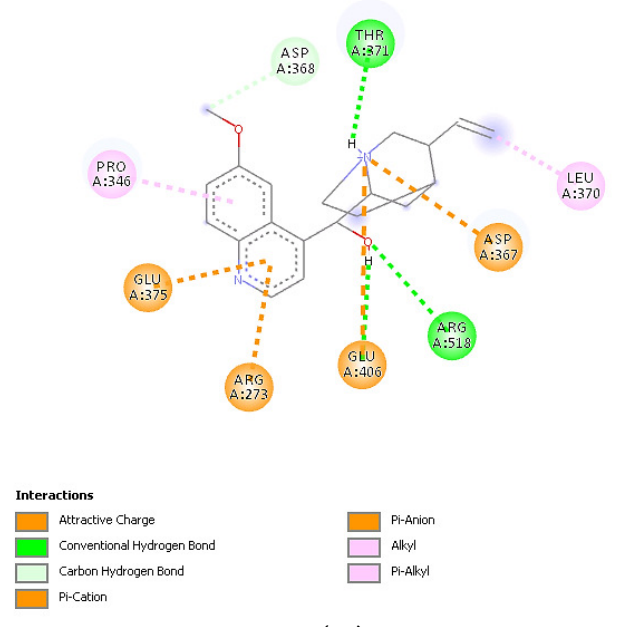

(C)

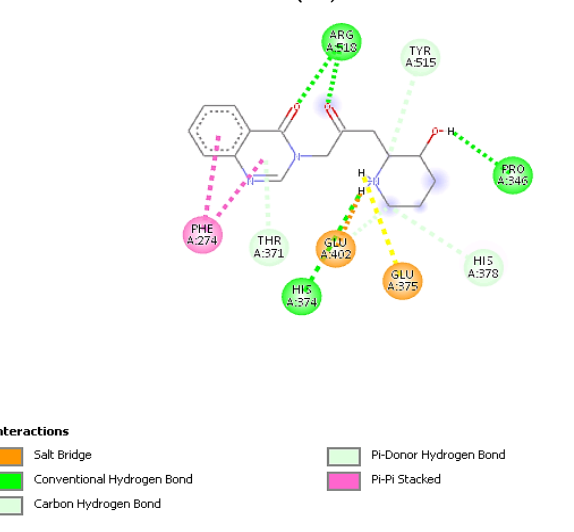

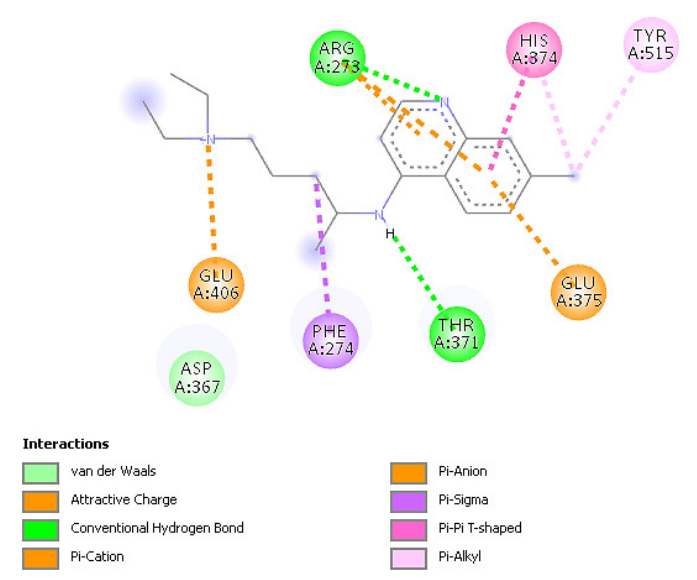

(B)

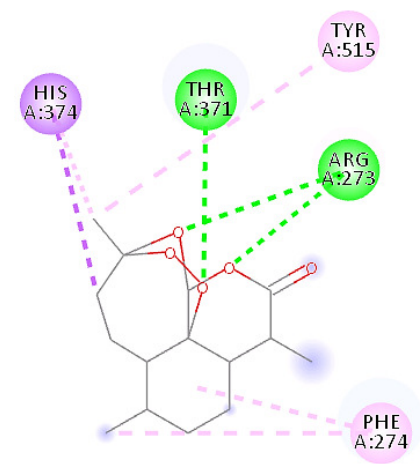

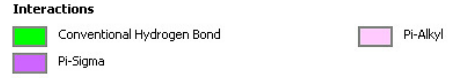

(D)

(E)

Figure 3. Depiction of 2D structures showing the interactions between the studied ligands and the receptor ACE2. A, Interactions between the reference molecule (XX5) and ACE2. B, Interactions between Chloroquine and ACE2. C, Interactions between quinine and ACE2. D, Interactions between artemisinin and ACE2. E, Interactions between febrifugine and ACE2 
Benslama O et al. (2021). Not Sci Biol 13(2):10835

Table 3. Drug-likeness properties of studied phytochemicals

\begin{tabular}{|c|c|c|c|c|c|c|c|c|c|c|}
\hline & Chloroquine & Quinine & Artemisinin & Febrifugine & Brusatol & Chaparrin & Glaucarubin & Sergeolide & Tehranolide & $\begin{array}{c}\text { Yingzhaosu } \\
\text { A }\end{array}$ \\
\hline $\mathrm{MW} \mathrm{g} / \mathrm{mol}$ & 319.18 & 324.18 & 282.15 & 301.14 & 520.19 & 380.18 & 495.23 & 504.16 & 298.14 & 270.18 \\
\hline $\log \mathrm{P}$ & 2.68 & 1.016 & 3.039 & -0.093 & 0.899 & 0.233 & 0.166 & 0.513 & 0.964 & 2.099 \\
\hline $\log S$ & -4.55 & -3.71 & -3.42 & -1.83 & -3.30 & -1.67 & -2.52 & -3.02 & -2.28 & -2.38 \\
\hline $\mathrm{A} \log \mathrm{P}$ & 0.044 & -0.769 & 0.801 & -1.885 & -0.394 & -1.312 & -2.137 & -1.63 & 0.258 & 1.622 \\
\hline HBA & 3 & 4 & 5 & 6 & 11 & 7 & 10 & 11 & 6 & 4 \\
\hline HBD & 1 & 1 & 0 & 2 & 3 & 4 & 5 & 2 & 2 & 2 \\
\hline TPSA $\left(\AA^{2}\right)$ & 27.63 & 45.06 & 53.99 & 82.0 & 165.89 & 116.45 & 162.98 & 154.89 & 85.22 & 58.92 \\
\hline AMR & 95.91 & 96.08 & 61.35 & 81.71 & 124.31 & 92.79 & 117.26 & 116.89 & 64.23 & 68.74 \\
\hline $\mathrm{nRB}$ & 8 & 4 & 0 & 4 & 5 & 0 & 4 & 4 & 0 & 3 \\
\hline n. Atom & 48 & 48 & 42 & 41 & 69 & 55 & 71 & 64 & 43 & 45 \\
\hline n. RigidB & 15 & 23 & 23 & 20 & 36 & 31 & 35 & 37 & 24 & 17 \\
\hline n. AromRing & 2 & 2 & 0 & 1 & 0 & 0 & 0 & 0 & 0 & 0 \\
\hline $\mathrm{nHB}$ & 4 & 5 & 5 & 8 & 14 & 11 & 15 & 13 & 8 & 6 \\
\hline Lipinski & Yes & Yes & Yes & Yes & No & Yes & Yes & No & Yes & Yes \\
\hline Ghose & Yes & Yes & Yes & Yes & No & Yes & No & No & Yes & Yes \\
\hline CMC 50 & Yes & No & No & No & No & No & No & No & No & No \\
\hline Veber & Yes & Yes & Yes & Yes & No & Yes & No & No & Yes & Yes \\
\hline MDDR & No & No & No & No & No & No & No & No & No & No \\
\hline BBB Likeness & Yes & Yes & Yes & Yes & No & No & No & No & Yes & Yes \\
\hline uwQED & Yes & Yes & Yes & Yes & Yes & Yes & Yes & Yes & Yes & Yes \\
\hline wQED & Yes & Yes & Yes & Yes & Yes & Yes & Yes & Yes & Yes & Yes \\
\hline Bioavailability & 0.55 & 0.55 & 0.55 & 0.55 & 0.17 & 0.55 & 0.55 & 0.17 & 0.55 & 0.55 \\
\hline
\end{tabular}

MW, Molecular weight. HBD, Hydrogen Bond Donor. HBA, Hydrogen Bond Acceptors. AMR, Atom Molar Refractivity. TPSA, Topological Polar Surface Area. nRB, number of Rotable Bond. nRigidB, number of Rigid Bond. nHB, number of Hydrogen Bond.

Table 4. ADMET properties of studied phytochemicals

\begin{tabular}{|c|c|c|c|c|c|c|c|c|c|c|c|}
\hline & Chloroquine & Quinine & Artemisinin & Febrifugine & Brusatol & Chaparrin & Glaucarubin & Sergeolide & $\begin{array}{c}\text { Tehrano } \\
\text { lide }\end{array}$ & $\begin{array}{c}\text { Yingzhaosu } \\
\text { A }\end{array}$ \\
\hline \multirow{10}{*}{ 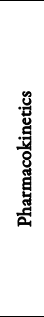 } & BBB & Yes & No & No & No & No & No & No & No & No & Yes \\
\hline & Caco2 & High & Medium & High & Medium & Medium & Medium & Medium & Medium & Medium & Low \\
\hline & PPB & $\begin{array}{l}\text { Weakly } \\
\text { bound }\end{array}$ & $\begin{array}{l}\text { Weakly } \\
\text { bound }\end{array}$ & $\begin{array}{l}\text { Weakly } \\
\text { bound }\end{array}$ & $\begin{array}{l}\text { Weakly } \\
\text { bound }\end{array}$ & $\begin{array}{l}\text { Weakly } \\
\text { bound }\end{array}$ & $\begin{array}{l}\text { Weakly } \\
\text { bound }\end{array}$ & $\begin{array}{l}\text { Weakly } \\
\text { bound }\end{array}$ & $\begin{array}{l}\text { Weakly } \\
\text { bound }\end{array}$ & $\begin{array}{l}\text { Weakly } \\
\text { bound }\end{array}$ & $\begin{array}{c}\text { Strongly } \\
\text { bound }\end{array}$ \\
\hline & HIA & High & High & High & High & High & Low & Moderate & Low & High & High \\
\hline & Pgp & No & Yes & No & No & Yes & Yes & Yes & Yes & Yes & No \\
\hline & CYP1A2 inhibitor & Yes & No & Yes & No & No & No & No & No & No & No \\
\hline & CYP2C19inhibitor & No & No & No & No & No & No & No & No & No & No \\
\hline & CYP2C9 inhibitor & No & Yes & No & No & No & No & No & No & No & No \\
\hline & CYP2D6 inhibitor & Yes & No & No & No & No & No & No & No & No & No \\
\hline & CYP3A4 inhibitor & Yes & No & No & No & No & No & No & No & No & No \\
\hline \multirow{5}{*}{ 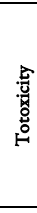 } & Ames test & Mutagen & Mutagen & Mutagen & Mutagen & NM & NM & NM & Mutagen & NM & NM \\
\hline & $\begin{array}{l}\text { Carcinogenicity } \\
\text { (Mouse) }\end{array}$ & Negative & Negative & Negative & Negative & Negative & Negative & Positive & Positive & Negative & Negative \\
\hline & $\begin{array}{c}\text { Carcinogenicity } \\
\text { (Rat) }\end{array}$ & Negative & Negative & Negative & Negative & Positive & Negative & Negative & Negative & Positive & Negative \\
\hline & hERG_inhibition & Medium risk & High risk & Low risk & Medium risk & $\begin{array}{c}\text { Medium } \\
\text { risk }\end{array}$ & Low risk & Ambiguous & $\begin{array}{c}\text { Medium } \\
\text { risk }\end{array}$ & Low risk & Low risk \\
\hline & H-HT & Positive & Positive & Negative & Positive & Positive & Negative & Positive & Positive & Positive & Negative \\
\hline & $\begin{array}{c}\text { Synthetic } \\
\text { accessibility }\end{array}$ & 2.76 & 9.22 & 6.13 & 3.23 & 6.50 & 6.02 & 6.80 & 6.68 & 5.65 & 6.00 \\
\hline
\end{tabular}

BBB, Blood-Brain Barrier. Pgp, P glycoprotein transport. PPB, Plasma Protein Binding. HIA, Human Intestinal Absorption. Caco2, Permeability assay. hERG, human Ether-a-go-go-Related Gene potassium channel. H-HT, Human Hepatotoxicity. NM, Non mutagen. 


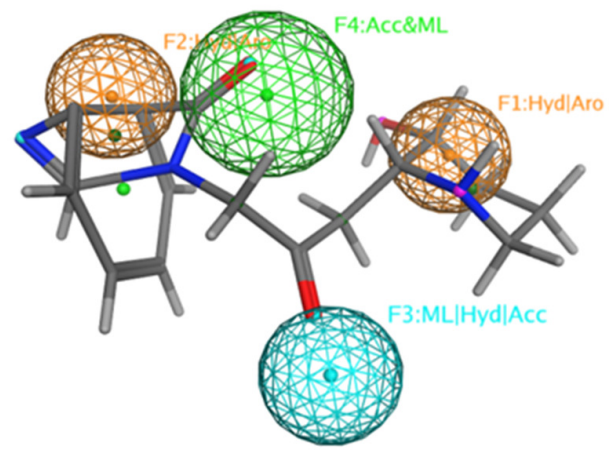

(A)

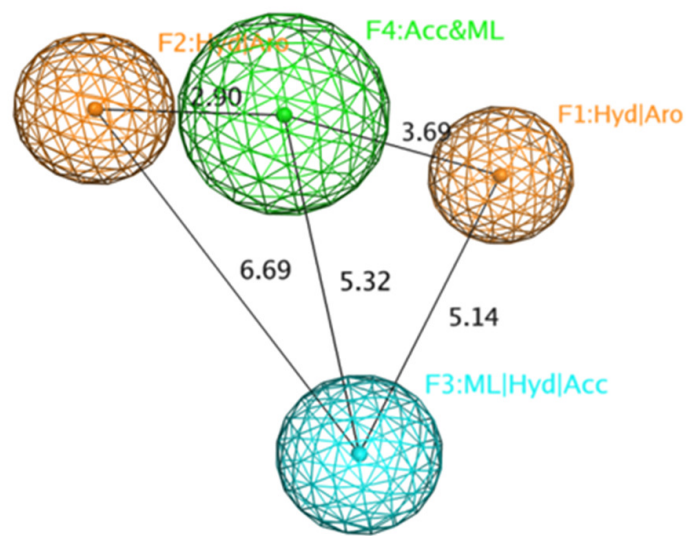

(C)

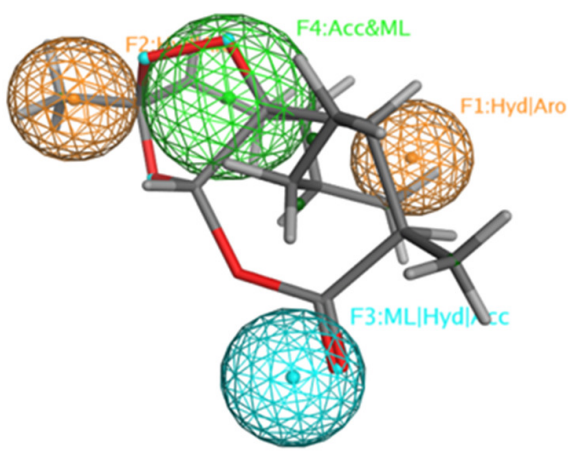

(B)

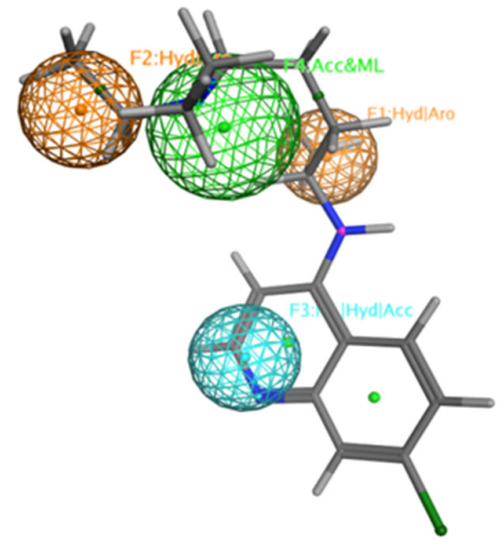

(D)

Figure 4. Pharmacophore model and its mapping to representative compounds. (A) The topological features of the model: the cyan contour represents metal ligator cum hydrophobic region cum hydrogen bond acceptor (ML/Hyd/Acc), green contour represents hydrogen bond acceptor cum metal ligator (ACC/ML), orange contour represents hydrophobic aromatic ring (Hyd/aro) features. (B) Chloroquine mapped with the model; (C) Febrifugine mapped with the model. (D) Artemisinin mapped with the model.

\section{Discussion}

\section{Molecular docking and interaction analysis}

From the results obtained from the molecular docking of the nine phytochemical molecules within the catalytic pocket of the ACE2 receptor and their comparison with the results found for the co-crystallized reference molecule XX5 and the chloroquine which is already used as a drug against COVID-19 infection, we noted that artemisinin, quinine and febrifugine are the most interesting antimalarial compounds in this study. In this group of molecules, Febrifugine has the best binding energy, that is lower than that of the reference molecule and that of chloroquine. This ligand also showed a number of interactions close to that of the reference molecule and superior to that of chloroquine. In addition, it reacts with the majority of key amino acids of ACE2 active site. On another side, artemisinin appears to be the closest ligand to chloroquine with almost equal binding energy and the same number of interactions. In addition, these ligands interact with the same key amino acids of the ACE2 binding site.

\section{Drug likness activity and ADMET study}

Lipinski has set up a set of characters to validate the bioavailability of a compound by the oral route based on four physicochemical properties (Lipinski et al., 1997). To bing therapeutically effective, a drug candidate must have a maximum molecular weight of $500 \mathrm{~g} / \mathrm{mol}$, so that it can be easily absorbed through the 
various biological membranes. It must have a maximum of 5 hydrogen bond donors, a maximum of 10 hydrogen bond acceptors and the decimal logarithm of the water partition coefficient noted logP, must be less than 5 .

With the exception of Brusatol and sergeolide, all the other studied molecules have a molecular weight of less than $500 \mathrm{~g} / \mathrm{mol}$. Like the chloroquine, the ligands quinine, artemisinin, febrifugine, chaparrin, glaucarubin, tehranolide and yingzhaosu A have respected the number of hydrogen bond donors which is less than or equal to 5. In addition, the number of hydrogen bond acceptors was also respected for all the tested ligands. The lipophilicity values presented by the $\log P$ measurement are all less than 5 , which suggests good absorption by the body. These molecules generally have a hydrophilic character, thus, they are mainly distributed in aqueous regions of the organism, such as blood serum, which facilitates their distribution. In conclusion of this first part of investigation, Lipinski's rule was correctly validated by chloroquine, quinine, artemisinin, febrifugine, chaparrin, glaucarubin, tehranolide and yingzhaosu A, but violated by Brusatol and Sergeoliden.

In addition to the Lipinski rule, seven other rules based on several criteria determining the potential pharmacokinetic quality of the molecules were analyzed to determine the drug-likeness of the different molecules. These rules are the Ghose rule (Ghose et al., 1999), CMC-50- Like rule (Ghose et al., 1999), Veber (Veber et al., 2002), MDDR-Like rule, BBB Likeness, and the quantitative estimate of drug-likeness (QED). The use of these filters refines the results by reducing the failure rate attributable to a pharmacokinetic cause, thus allowing the selection of the molecules most apt to become drug candidates. From the results mentioned in Table 3, it is obvious that chloroquine is the best molecule when it comes to the verification of rules, where with the exception of the MDDR-like rule, no violation is noted. In addition to the MDDR-Like rule, the CMC-50- Like rule was violated for Quinine, artemisinin, febrifugine, glaucarubin, tehranolide and yingzhaosu A. Chaparrin and brusatol gave unsatisfactory results by the violation of the majority of the rules.

Another important criterion introduced by Veber is the topological polar surface area (TPSA), which must be less than $140 \AA^{2}$. This rule is verified for all compounds except for glaucarubin and brusatol. In fact, the TPSA predicts intestinal absorption and the passage of the blood brain barrier which is difficult for a compound with a TPAS at $140 \AA^{2}$ (Cecchelli et al., 2007). The number of rotational links is another crucial criterion of the Veber rule. For a good oral bioavailability, this factor must be less than 10, which is verified for all the compounds studied.

The criterion of atom molar refractivity (AMR) which must be included between 40 and 130, is also verified for all the studied ligands. In addition, different estimates have been made for the water solubility, which is expressed by the $\log S$ measurement. For chloroquine a value of -4.55 elucidates a moderate solubility. When for quinine, artemisinin, brusatol, tehranolide, glaucarubin, sergeoliden and yingzhaosu A $\log S$ was between -4 and -2 , they are therefore qualified as soluble. While, febrifugine and chaparrin are considered as very soluble molecules with the values of -1.83 and -1.67 , respectively. Typically, a good solubility goes along with a good absorption and distribution in the organism, which makes the molecule a good therapeutic candidate. The bioavailability is a subcategory of absorption that corresponds to the importance of the passage of the drug in the general circulation, thus gaining the site of action ( $\mathrm{Sim}, 2015)$. With the exception of brusatol and sergeolide, all other molecules show a good bioavailability score of 0.55 .

A criterion of major importance is to study the potential interaction with the P glycoprotein (Pgp) which is a membrane transporter involved in the efflux of drugs, and therefore in their pharmacokinetic quality. Pgp influences the bioavailability of many drugs, by pumping them from the digestive tract, which will ultimately make them less effective. Chloroquine, artemisinin, febrifugine and yingzhaosu A are not effluent by $\mathrm{P}$ glycoprotein, they are then bioavailable, unlike that quinine, brusatol, tehranolide, glaucarubin, chaparrin and sergeoliden are easily effluent through this pump system. Likewise, the analysis of the binding to cytochromes $\mathrm{P} 450$, which intervenes in the metabolism of drugs, is another criterion providing information on the ADMET properties of the molecule. Chloroquine is capable of inhibiting three families of $\mathrm{P} 450$ cytochromes which are CYP1A2, CYP2D6 and CYP3A4. While quinine and artemisinin, each of them 
inhibits only one family that is CYP2C9 and CYP1A2, respectively. On the other hand, febrifugine, brusatol, chaparrin, tehranolide, glaucarubin, sergeoliden and yingzhaosu A, are not considered as inhibitors for the different families of $\mathrm{P} 450$ cytochromes. In fact, inhibitors can increase the plasma concentrations of certain drugs by slowing their metabolism, which can cause undesirable effects of these drugs with sometimes serious consequences (Vermeulen, 2003).

The permeability of biological membranes is an important pharmacological criterion for the evaluation of the therapeutic efficacy of a given molecule. This is expressed by the Caco-2 and Blood-brain barrier permeability (BBB) values. The permeability estimated by Caco- 2 is the most important for Chloroquine and artemisinin and less important for the other molecules. With the exception of chloroquine and yingzhaosu $\mathrm{A}$, all the other molecules cannot cross the blood-brain barrier, which is a favorable point for a drug candidate intended for a treatment against COVID-19, which typically must not reach the central nervous system. Another important pharmacological parameter is drug absorption. In fact, absorption is the process by which the drug passes into the general circulation from its site of administration. When administered orally, the active substance arrives in the intestine before being absorbed to join the bloodstream to be distributed throughout the body to exert its action in the target site. Effective absorption through the intestinal wall is therefore an important factor in the bioavailability of the drug. This factor is expressed by the HIA (Human intestinal absorption) value. The found HIA values show a high intestinal absorption for most of the studied molecules. The plasma protein binding (PPB) is another pharmacological criterion of crucial interest in the choice of therapeutically effective molecules. With the exception of yingzhaosu A, all the studied molecules are weakly bound to plasma proteins, which is an advantageous quality for these molecules because, generally, only the free fraction of the drug that is dissolved in the plasma is diffusible and can then reach the target tissues. But unlike, significant fixation of the drug by plasma proteins limits its bioavailability by making it less effective (Tillement et al., 2006).

Because the toxicity of drug candidates is the biggest cause of drug development failure, the studied molecules are also tested for their toxicological properties. Among the most important toxicity characteristics, mutagenicity, carcinogenicity, cardiotoxicity and hepatotoxicity (Toropov et al., 2014). For carcinogenicity, most compounds show a negative result for carcinogenicity tests in mouse and rat, while a positive response was detected in mouse for glaucarubin and sergeoliden, and in rat for Brusatol and tehranolide. Mutagenicity is also attributed to certain molecules. Moreover, cardiotoxicity is based on the assessment of inhibition of the hERG potassium channel, which can lead to cardiac arrest (Sanguinetti and Tristani-Firouzi, 2006). Chloroquine, febrifugine, brusatol and sergeoliden are classified as having a medium risk. On the other hand, artemisinin, chaparrin, tehranolide, glaucarubin and yingzhaosu A have a low risk. Whereas quinine seems to be a high-risk molecule. Hepatotoxicity is another important parameter to screen for a drug candidate. Artemisinin, brusatol, chaparrin, tehranolide and glaucarubin seem to pose no risk of injury or liver damage. Unlike that, a risk of hepatotoxicity is assimilated to chloroquine, quinine, febrifugine, sergeoliden and yingzhaosu A.

Synthetic accessibility is a criterion related to the medicinal chemistry which provides information on the ease of synthesis of a drug from the active molecule. The characteristic values of this factor are between 1 that indicating an easy synthesis, and 10 that indicating a difficult synthesis. According to the results mentioned in Table 4, Chloroquine and febrifugine are the easiest molecules to handle in pharmaceutical chemistry.

By analyzing the results of this section, it becomes clear that febrifugine and artemisinin are the most satisfactory phytochemicals with regard to their drug-likness and pharmacokinetic properties. The two molecules verified six of the eight studied rules. Like Chloroquine, the two molecules gave good results for the TPSA, number of rotational links, AMR, Pgp, HIA and PPB, thus verifying the bioavailability criterion. Regarding solubility, febrifugine records the best estimate in comparison with chloroquine and artemisinin. Unlike chloroquine, both molecules are BBB negative which is more advantageous for a therapeutic molecule not intended for the central nervous system. Caco2 values are better for chloroquine and artemisinin and medium for febrifugine. The ability to inhibit cytochrome P450 makes the two molecules safer than 
chloroquine in terms of blocking their metabolism and thus their efficient excretion from the body. On the toxicity side, artemisinin is qualified as a low cardio toxicological risk agent, while chloroquine and febrifugine have a medium risk. In addition, a risk of hepatotoxicity is attributed to chloroquine and febrifugine but not to artemisinin.

\section{Ligand-based pharmacophore modeling}

A total of 17 flexible alignments were generated from the training set, the alignment with the best quality in terms of similarity of the molecular features and the potential energy of the molecules was chosen for the construction of the pharmacophore model. The pharmacophoretic selected features are one metal ligator cum hydrophobic region cum hydrogen bond acceptor, one hydrogen bond acceptor cum metal ligator and one hydrophobic aromatic ring. The distances between the functional groups of the pharmacophore were calculated (Figure 4). A test set comprising Chloroquine, artemisinin and febrifugine was used for a pharmacophore search request using the created model. The result of the search gave 136 possible entries. Each entry represents a match of the pharmacophore query to one conformation of these three ligands. Only the most suitable conformations to the pharmacophore query (having the lowest RMSD) for the three molecules were chosen (Figure 4). This pharmacophore model can be used for the screening of molecule set for their inhibitory activity against the ACE2 receptor.

\section{Conclusions}

In this study, we have performed a molecular docking of a set of nine phytochemicals with the receptor ACE2, necessary for the binding of the SARS-Cov-2. Since chloroquine, which is an antimalarial, is today the only treatment for COVID-19 infection, the phytochemicals that were the subject of this study were chosen because they are known to have an antimalarial activity, therefore have the same therapeutic effect of chloroquine. On the basis of the values of the binding energies and the number of interactions obtained after the docking process of the molecules, and in comparison, with the results obtained from the co-crystallized reference molecule, the ligands which have the most affinity with the ACE2 receptor are chloroquine, quinine, artemisinin and febrifugine. The latter is remarkably the closest ligand to the reference molecule, and Artemisinin has showed results closest to those of chloroquine. In a second part of this study, the chosen phytochemical molecules have been subject to drug-likness analysis and ADMET test. According to the results obtained, it seems that in addition to chloroquine, the ligands febrifugine and artemisinin are the most relevant phytochemicals. These two molecules have verified almost all the chosen filters for the drug-likness analysis and have shown pharmacokinetic properties of satisfactory quality. In addition to their bioavailability, febrifugine and artemisinin do not appear to be highly toxic to the body, thus qualifying them as good candidate drugs. With their favourable properties, febrifugine and artemisinin deserve further investigation as a possible treatment of COVID-19 especially in-vitro and in-vivo. We have also established a pharmacophore model using a set of molecules comprising the most relevant ligands of this study namely the reference molecule XX5, chloroquine, quinine, artemisinin and febrifugine. The common pharmacophoric features to these ligands were chosen to build the pharmacophore model. These features are one metal ligator cum hydrophobic region cum hydrogen bond acceptor, one hydrogen bond acceptor cum metal ligator and one hydrophobic aromatic ring. In addition, the distances between the functional groups of the pharmacophore were calculated. This pharmacophore model is proposed for the screening of a set of molecules in order to discover potential inhibitors of the ACE2 receptor. 


\section{Authors' Contributions}

O.B. took the lead in the data curation, formal analysis, funding acquisition, methodology, resources, software, supervision, validation. N.M. and R.A. provided critical feedback and helped shape the research, analysis and manuscript, visualisation, writing review and editing. All authors read and approved the final manuscript.

\section{Acknowledgements}

This research received no specific grant from any funding agency in the public, commercial, or not-forprofit sectors.

\section{Conflict of Interests}

The authors declare that there are no conflicts of interest related to this article.

\section{References}

Aanouz I, Belhassan A, Khatabi KE, Lakhlifi T, Idrissi ME, Bouachrine M (2020). Moroccan medicinal plants as inhibitors of COVID-19: Computational investigations. Journal of Biomolecular Structure and Dynamics 2020:1-12. https://doi.org/10.1080/07391102.2020.1758790

Abdelli I, Hassani F, Bekkel Brikci D, Ghalem S (2020). In silico study the inhibition of angiotensin converting enzyme 2 receptor of COVID-19 by Ammoides verticillata components harvested from Western Algeria. Journal of Biomolecular Structure and Dynamics 2020:1-17. https://doi.org/10.1080/07391102.2020.1763199

Bruce-Chwatt LJ (1981). Chemotherapy of malaria. WHO (2nd ed), Geneva, Switzerland.

Cecchelli R, Berezowski V, Lundquist S, Culot M, Renftel M, Dehouck MP, Fenart L (2007). Modelling of the bloodbrain barrier in drug discovery and development. Nature Reviews Drug Discovery 6(8):650-661. https://doi.org/10.1038/nrd2368

Di Trani L, Savarino A, Campitelli L, Norelli S, Puzelli S, D’Ostilio D, ... Cassone A (2007). Different pH requirements are associated with divergent inhibitory effects of chloroquine on human and avian influenza A viruses. Virology Journal 4:39. https://doi.org/10.1186/1743-422X-4-39

Dong E, Du H, Gardner L (2020). An interactive web-based dashboard to track COVID-19 in 447 real time. The Lancet Infectious Disease 20(5):P533-534. https://doi.org/10.1016/S1473-448 3099(20)30120-1

Foley M, Tilley L (1998). Quinoline antimalarials: mechanisms of action and resistance and prospects for new agents. Pharmacology \& Therapeutics 79(1):55-87. https://doi.org/10.1016/s0163-7258(98)00012-6

Gautreta P, Lagiera J-C, Parolaa P, Hoang VT, Meddeb L, Mailhe M, ... Raoult D (2000). Hydroxychloroquine and azithromycin as a treatment of COVID-19: results of an openlabel non-randomized clinical trial. International Journal of Antimicrobial Agents 56(1):105949. https://doi.org/10.1016/j.ijantimicag.2020.105949

Ghose AK, Viswanadhan VN, Wendoloski JJ (1999). A knowledge-based approach in designing combinatorial or medicinal chemistry libraries for drug discovery. 1. A qualitative and quantitative characterization of known drug databases. Journal of Combinatorial Chemistry 1:55. https://doi.org/10.1021/cc9800071

Gorbalenya AE, Baker SC, Baric RS, de Groot RJ, Drosten C, Gulyaeva AA, ... Ziebuhr J (2020). Severe acute respiratory syndrome-related coronavirus: the species and its viruses-a 4 statement of the Coronavirus Study Group. Nature Microbiology. https://doi.org/10.1101/2020.02.07.937862

Huang J, Chen H, Shang Y, Zhu H, Chen G, Chen Y, ... Xia J (2020). Efficacy of chloroquine and Lopinavir/ Ritonavir in mild/general novel coronavirus (CoVID-19) infections: a prospective, open-label, multicenter randomized controlled clinical study. Trials 21:1. https://doi.org/10.21203/rs.3.rs-16392/v1 
Joshi T, Sharma P, Mathpal S, Pundir H, Bhatt V, Chandra S (2020). In silico screening of natural compounds against COVID-19 by targeting Mpro and ACE2 using molecular docking. European Review for Medical and Pharmacological Sciences 24:4529-4536. https://doi.org/10.26355/eurrev_202004_21036

Krafts K, Hempelmann E, Skórska-Stania A (2010). From methylene blue to chloroquine: a brief review of the development of an antimalarial therapy. Parasitology Research 111:1-6. https://doi.org/10.1007/s00436-0122886- $x$

Kumar A, Choudhir G, Shukla SK, Sharma M, Tyagi P, Bhushan A, Rathore M (2020). Identification of phytochemical inhibitors against main protease of COVID-19 using molecular modeling approaches. Journal of Biomolecular Structure and Dynamics https://doi.org/10.1080/07391102.2020.1772112

Kuznik A, Bencina M, Svajger U, Jeras M, Rozman B, Jerala R (2011). Mechanism of endosomal TLR Inhibition by Antimalarial drugs and imidazoquinolines. Journal of Immunology 186:4794-804. https://doi.org/10.4049/jimmunol.1000702

Lipinski CA, Lombardo F, Dominy BW, Feeney PJ (1997). Experimental and computational approaches to estimate solubility and permeabilityin drug discovery and development settings. Advanced Drug Delivery Reviews 46(13):3-26. https://doi.org/10.1016/S0169-409X(96)00423-1

Macfarlane DE, Manzel L (1998). Antagonism of immunostimulatory CpG-oligodeoxynucleotides by quinacrine, chloroquine, and structurally related compounds. Journal of Immunology 160(3):1122-31.

Marmor MF, Kellner U, Lai TY, Melles RB, Mieler WF (2016). Recommendations on screening for chloroquine and hydroxychloroquine retinopathy (2016 Revision). American Academy of Ophthalmology 123(6):1386-94. https://doi.org/10.1016/j.ophtha.2016.01.058

Meng X-Y, Zhang H-X, Mezei Z, Cui M (2011). Molecular docking: A powerful approach for structure-based drug discovery. Current Computer-Aided Drug Design 7(2):146-157. https://doi.org/10.2174/157340911795677602

Mojab F (2012). Antimalarial natural products: a review. Avicenna Journal of Phytomedicine 2(2):52-62.

Sanguinetti MC, Tristani-Firouzi M (2006). hERG potassium channels and cardiac arrhythmia. Nature 440:463-469. https://doi.org/10.1038/nature04710

Savarino A, Gennero L, Sperber K, Boelaert JR (2001). The anti-HIV-1 activity of chloroquine. Journal of Clinical Virology 20:131-5. https://doi.org/10.1016/s1386-6532(00)00139-6

Sim DSM (2015). Drug Absorption and Bioavailability. In: Chan Y, Ng K, Sim D (Eds). Pharmacological Basis of Acute Care. Springer, Cham. pp 17-26. https://doi.org/10.1007/978-3-319-10386-0_3

Tai W, Lu T, Yuan H, Wang F, Liu H, Lu S, ... Chen Y (2012). Pharmacophore modeling and virtual screening studies to identify new c-Met inhibitors. Journal of Molecular Modeling 18(7):3087-3100. https://doi.org/10.1007/s00894-011-1328-5

Tillement JP, Duché JD, Barré J (2006). Liaisons des médicaments aux protéines circulantes: caractéristiques, rôles et modifications physio-pathologiques [Drug binding to blood proteins: characteristics, roles and pathophysiological changes]. Bulletin de l'Académie Nationale de Médecine 190(4-5):935-947.

Toropov AA, Toropova AP, Raska Jr I, Leszczynska D, Leszczynski J (2014). Comprehension of drug toxicity: Software and databases. Computers in Biology and Medicine 45:20-25. https://doi.org/10.1016/j.compbiomed.2013.11.013

Veber DF, Johnson SR, Cheng HY, Smith BR, Ward KW, Kopple KD (2002). Molecular properties that influence the oral bioavailability of drug candidates. Journal of Medicinal Chemistry 45(12):2615-2623. https://doi.org/10.1021/jm020017n

Vermeulen NPE (2003). Prediction of drug metabolism: the case of cytochrome P450 2D6. Current Topics in Medicinal Chemistry 3(11):1227-1239. https://doi.org/10.2174/1568026033451998

Vincent MJ, Bergeron E, Benjannet S, Erickson BR, Rollin PE, Ksiazek TG, ... Nichol ST (2005). Chloroquine is a potent inhibitor of SARS coronavirus infection and spread. Virology Journal 2:69. https://doi.org/10.1186/1743- $422 \mathrm{X}$ 2- 69

Vincent MJ, Bergeron E, Benjannet S, Erickson BR, Rollin PE, Ksiazek TG (2005). Chloroquine is a potent inhibitor of SARS coronavirus infection and spread. Virology Journal 2:69. https://doi.org/10.1186/1743-422X-2-69

Wang M, Cao R, Zhang L, Yang X, Liu J, Xu X, ... Xiao G (2020). Remdesivir and chloroquine effectively inhibit the recently emerged novel coronavirus (2019-nCoV) in vitro. Cell Research 30:269-271. https://doi.org/10.1038/s41422-020-0282-0 
Yao X, Ye F, Zhang M, Cui C, Huang B, Niu P, ... Liu D (2020). Hydroxychloroquine for the Treatment of Severe Acute Respiratory Syndrome Coronavirus 2 (SARS-CoV-2). Clinical Infectious Diseases 71(15):732-739. https://doi.org/10.1093/cid/ciaa237

OPEN ACCESS (c) (1)
The journal offers free, immediate, and unrestricted access to peer-reviewed research and scholarly work. Users are allowed to read, download, copy, distribute, print, search, or link to the full texts of the articles, or use them for any other lawful purpose, without asking prior permission from the publisher or the author.

License - Articles published in Notulae Scientia Biologicae are Open-Access, distributed under the terms and conditions of the Creative Commons Attribution (CC BY 4.0) License.

(c) Articles by the authors; SHST, Cluj-Napoca, Romania. The journal allows the author(s) to hold the copyright/to retain publishing rights without restriction. 